\title{
Painless Posterior Scleritis: A Rare Presentation
}

\author{
Summaya Khan, Mahum Faheem, Imran Basit and Ammarah Ashraf \\ Department of Ophthalmology, Armed Forces Institute of Ophthalmology, Rawalpindi, Pakistan
}

\begin{abstract}
Posterior scleritis is an underdiagnosed condition due to its rarity and is frequently misdiagnosed due to its varied presentation. ${ }^{1}$ Therefore, it is imperative to know that it can present clinically as exudative retinal detachment, proptosis, disc oedema, choroidal folds, subretinal mass, and myositis.

We report a case of 48-year female who presented with gradual, progressive loss of vision in left eye. There were multiple exudative retinal detachments with choroidal folds and disc oedema. Ultrasonography (USG) B scan and contrast enhanced magnetic resonance imaging (MRI) orbit revealed the classic T-sign. With oral steroids, she improved clinically. Posterior scleritis is a painful condition, but the patient did not report any pain in or around the globe, which makes this case a unique experience.
\end{abstract}

Key Words: Posterior scleritis, Exudative retinal detachment, T-sign.

How to cite this article: Khan S, Faheem M, Basit I, Ashraf A. Painless Posterior Scleritis: A Rare Presentation. J Coll Physicians Surg Pak 2021; 31(05):594-595.

\section{INTRODUCTION}

Posterior scleritis is labelled when sclera is inflamed beyond the equator. ${ }^{1,2}$ It is both a potentially blinding and painful condition, which can be associated with systemic disorders. It has also been reported to occur secondary to vasculitis associated with rheumatoid arthritis. ${ }^{3}$ Posterior scleritis is difficult to diagnose, requiring good knowledge of the disease and treatment is variable. ${ }^{4}$

Here, we report a rare presentation of posterior scleritis in a 48year female, who presented with unilateral decrease in vision, but with no pain. Therefore, this case needs to be highlighted.

\section{CASE REPORT}

A 48-year female presented at Armed Forces Institute of Ophthalmology with complain of gradual, progressive loss of vision in lefteye for the lastone week. Ophthalmologic examination showed an unaided visual acuity of LogMAR $0.0(6 / 6)$ in right eye, while hand movements in left eye. There was relative afferent pupillary defect (RAPD) in lefteye, while rest of theanterior segment examination of both eyes was non-contributory. Posterior segment examination of left eye revealed optic disc oedema, choroidal folds and multiple exudative retinal detachments (Figure 1).

Correspondence to: Dr. Mahum Faheem, Department of Ophthalmology, Armed Forces Institute of Ophthalmology, Rawalpindi, Pakistan

E-mail: mahumfaheem23@gmail.com

Received: September 12, 2019; Revised: November 20, 2019; Accepted: November 28, 2019

DOI: https://doi.org/10.29271/jcpsp.2021.05.594

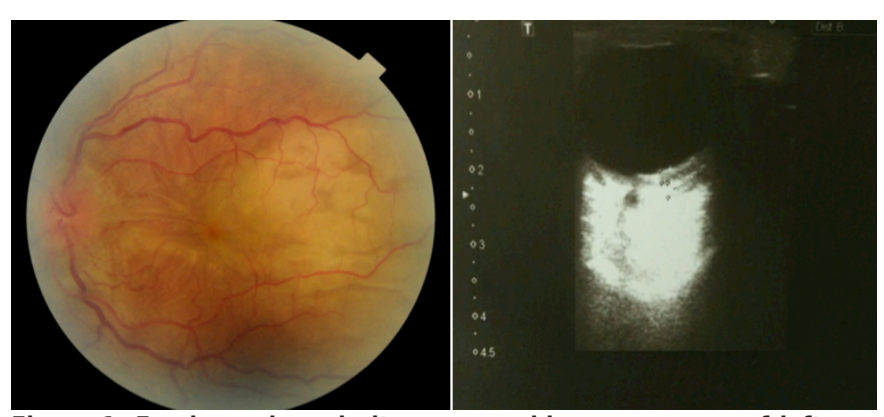

Figure 1: Fundoscopic and ultrasonographic appearances of left eye before treatment.

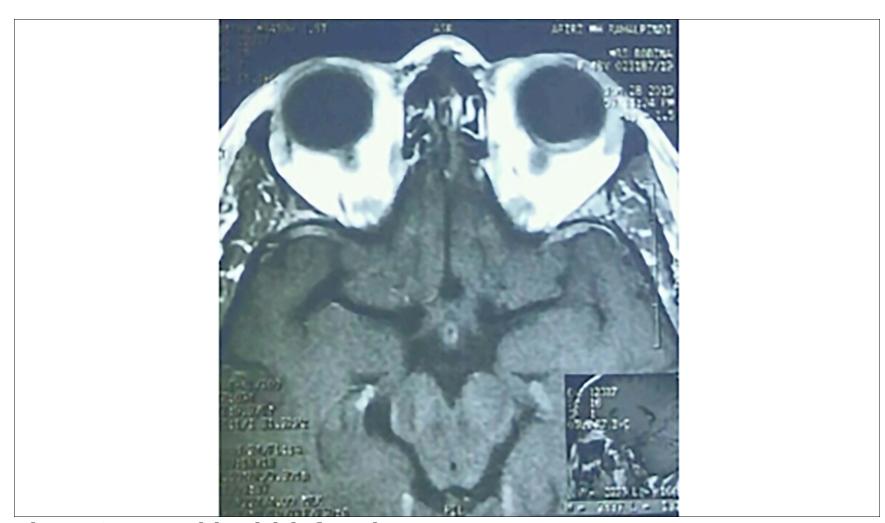

Figure 2: MRI orbit with left T-sign.

Complete blood count, C-reactive protein, anti-nuclear antibodies, anti-cardiolipin antibodies, serology for TORCH, and VDRL were normal. Mantoux test was negative. Chest X-ray and angiotensin conversion enzyme (ACE) assay was normal. However, her rheumatoid factor was positive and erythrocyte sedimentation rate was mildly raised.

B-mode ultrasound of left eye showed fluid beneath Tenon's capsule (characteristic 'T-sign'). This 'T-sign' was also depicted 
in her left globe on contrast-enhanced T1-weighted MRI brain (Figure 2). A diagnosis of posterior scleritis was made; and she was treated with oral prednisolone $1 \mathrm{mg} / \mathrm{kg} /$ day and topical nonsteroidal anti-inflammatory drugs (NSAIDS). On $5^{\text {th }}$ follow-up day, clinical examination revealed visual improvement from hand movement to LogMAR 0.6_(6/24) and significant improvement in optic disc oedema of her left eye, with moderate resolution of subretinal fluid.

Follow-up after two weeks showed visual acuity of LogMAR 0.0 $(6 / 6)$ and complete resolution of optic disc oedema and subretinal exudation in left eye (Figure 3). B-mode ultrasound of left eye demonstrated resolution of fluid beneath Tenon's capsule.

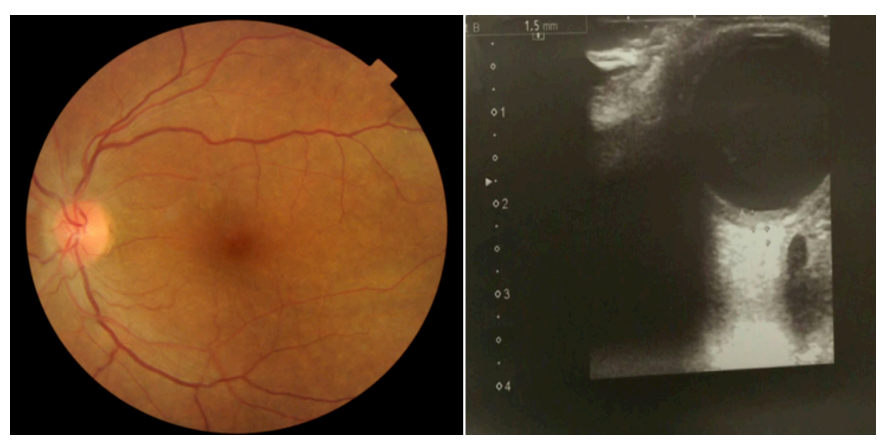

Figure 3: Fundoscopic and ultrasonographic appearances of left eye after treatment.

\section{DISCUSSION}

Posterior scleritis is a painful blinding condition, which has been reported to account for $2 \%-12 \%$ of all the cases of scleritis. ${ }^{5} \mathrm{~A}$ world-wide increase in incidence of posterior scleritis has been noted due to increased availability of modern imaging tools. It has also been reported in pediatric age group, often in the absence of any systemic association. ${ }^{6}$ Clinically, posterior scleritis can present with a spectrum of manifestations; exudative retinal detachment, uveal effusion, disc oedema and choroidal folds are most commonly seen. However, literature suggests that posterior scleritis could even mimic a choroidal tumor. ${ }^{7}$ Hence, it remains a diagnostic challenge to differentiate it from achoroidal lesion. Posterior scleritis can be well managed if diagnosed in time; otherwise, it can lead to permanent visual loss.

This patient had sudden painless deterioration of vision with unilateral disc oedema and exudative retinal detachment. As these signs are associated with multiple systemic disorders, so often the possibility of posterior scleritis were overlooked. The best approach in such cases would be to perform urgent B-mode ultrasound so that this blinding condition is diagnosed in time.

The peculiarity of this case was the painless presentation. Posterior scleritis has been labelled painful condition in the literature, so we included neuroretinitis in the differential diagnosis was included. But there were no vitritis, macular exudates and other supporting evidence, which highlights that posterior scleritis is a diagnostic challenge.

Posterior scleritis is a rare entity, in which patients can complain of fluctuating symptoms. Initiation of aggressive therapy can help prevent devastating complications. ${ }^{8}$ Therefore, timely diagnosis and treatment are vital in rescuing patient's eye.

\section{PATIENT'S CONSENT:}

Informed consent was taken from the patient.

\section{CONFLICT OF INTEREST:}

The authors declared no conflict of interest.

\section{AUTHORS' CONTRIBUTION:}

SK: Substantial contributions to the concept or design of the work; acquisition; analysis and data interpretation.

MF: Drafting the work; revising it critically for important intellectual content.

IB: Final approval of version to be published.

AA: Ensuring that questions related to work accuracy or integrity are appropriately resolved.

\section{REFERENCES}

1. Liu AT, Luk FO, Chan CK. A case of giant nodular posterior scleritis mimicking choroidal malignancy. Indian J Ophthalmol 2015; 63(12):919-21. doi: 10.4103/0301-4738. 176038.

2. Kumar A, Ghose A, Biswas J, Majumder PD. Clinical profile of patients with posterior scleritis: A report from Eastern India. Indian J Ophthalmol 2018; 66:1109-12. doi: 10.4103/ijo. IJO_121_18.

3. Scott DL, Wolfe F, Huizinga TW. Rheumatoid arthritis. Lancet 2010; 376(9746):1094-108. doi: 10.1016/ S0140-6736(10)60826-4.

4. Shenoy R1, Suryawanshi M1, Isaac R1, Philip SK. Posterior scleritis in pediatric age group: A case report and review of literature. Oman J Ophthalmol 2016; 9:1:59-62. doi: 10.4103/0974-620X.176121.

5. Lavric A, Gonzalez-Lopez JJ, Majumder PD, Bansal N, Biswas J, Pavesio C, et al. Posterior Scleritis: Analysis of Epidemiology, Clinical Factors, and Risk of Recurrence in a Cohort of 114 Patients. Ocul Immunol Inflamm 2016; 24(1): 6-15. doi: 10.3109/09273948.2015.1005240.

6. Erdol H, Kola M, Turk A. Optical coherence tomography findings in a child with posterior scleritis. Eur J Ophthalmol 2008; 18:1007-10. doi: 10.1177/112067210801800625.

7. Hage R, Jean-Charles A, Guyomarch J, Rahimian O, Donnio A, Merle H. Nodular posterior scleritis mimicking choroidal metastasis: A report of two cases. Clin Ophthalmol 2011; 5:877-80. doi: 10.2147/OPTH.S21255.

8. Keino H, Watanabe T, Taki W, Nakashima C, Okada AA. Clinical features and visual outcomes of Japanese patients with scleritis. Br J Ophthalmol 2010; 94:1459-63. doi: 10.1136/ bjo.2009.171744. 